\title{
Aszitespunktion: Abdominale Parazentese
}

Bosshard, S ; Mullhaupt, B

DOI: https://doi.org/10.1024/1661-8157/a000954

Other titles: Puncture of ascites: abdominal paracentesis

Posted at the Zurich Open Repository and Archive, University of Zurich ZORA URL: https://doi.org/10.5167/uzh-67471

Journal Article

Accepted Version

Originally published at:

Bosshard, S; Mullhaupt, B (2012). Aszitespunktion: Abdominale Parazentese. Praxis, 101(12):757-766. DOI: https://doi.org/10.1024/1661-8157/a000954 
DOI 10.1024/1661-8157/a000954

((PX12_Rubrik CME))

\title{
Aszitespunktion: Abdominale Parazentese
}

\author{
Abkürzungen \\ PMN Polymorphkernige Zellen \\ SAAG Serum-Aszites- Albumin-Gradient \\ SBP Spontanbakterielle Peritonitis
}

Definition ((Ü1))

Der Terminus Aszites stammt vom griechischen Wort Askites (aбkítns; Wassersucht) ab und meint die pathologische Flüssigkeitsansammlung im freien Peritonealraum. Aszites ist meist keine eigene Störung, sondern fast immer ein Symptom einer zugrundeliegenden Erkrankung. Die weitaus häufigste Ursache von Aszites ist die Leberzirrhose. Umgekehrt ist Aszites, zusammen mit dem hepatozellulären Karzinom, auch die häufigste Komplikation bei einer Leberzirrhose [1]. Der folgende Artikel handelt von der abdominalen Parazentese mit Augenmerk auf die Indikation und Durchführung der Punktion resp. Drainage und die Analyse des Aszites. 
Klinik ((Ü1))

Aszites äussert sich klinisch durch eine Vorwölbung des Bauches. Im Unterschied zu Meteorismus als Ursache für die Bauchvergrösserung, verteilt sich der Aszites aufgrund des höheren Gewichts gegenüber den meteoristischen Darmschlingen möglichst breit und führt beim liegenden Patienten zu einer starken Vorwölbung der Flanken. Geübte können Aszites klinisch durch Perkussion ab einer Menge von einem Liter nachweisen. Die gasgefüllten Darmschlingen «schwimmen» auf der Flüssigkeit und verursachen einen tympanischen Klopfschall, während der Klopfschall über der Flüssigkeit gedämpft ist (Abb. 1). Bei Lagerung zur Seite hin verschiebt sich die Klopfschallgrenze entsprechend der Verschiebung des Aszites durch die Schwerkraft (Abb. 2). Wesentlich sensitiver ist der Aszitesnachweis mittels Ultraschall. Da die Ursache von Aszites vielfältig ist, sollte als erster diagnostischer Schritt eine Aszitespunktion durchgeführt werden. Die häufigsten Ursachen von Aszites sind in Tabelle 1 zusammengefasst.

((etwa hier Abb. 1 und 2))

Abb. 1: Patient in Rückenlage. Klopfschalldämpfung markiert durch gestreifte Fläche.

Abb. 2: Verschiebung der Klopfschallgrenze nach medial hin bei Lagerung des Patienten nach rechts

((etwa hier Tab. 1))

Eine gefürchtete Komplikation bei Patienten mit einer Leberzirrhose und Aszites ist die spontanbakterielle Peritonitis (SBP). Patienten mit SBP haben entweder keine oder typischerweise nur leichte, unspezifische Beschwerden wie Nausea, Fieber, Unwohlsein oder Verwirrtheit, selten Bauchschmerzen. Das Auftreten einer SBP ist ein prognostisch schlechtes Zeichen und geht mit hoher Mortalität einher [2]. 


\section{Abklärungsstrategie ((Ü1))}

\section{Allgemein ((Ü2))}

Eine Aszitesanalyse lässt Rückschlüsse auf die Ursache des Aszites zu und sollte bei neu diagnostiziertem Aszites immer durchgeführt werden. Der häufigste Grund für eine Aszitespunktion bei Patienten mit bekannter Leberzirrhose und Aszites ist der Ausschluss einer spontanbakteriellen Peritonitis [3] oder die Behandlung eines therapierefraktären Aszites. Obwohl kein absoluter Grenzwert besteht, wird eine präinterventionelle Fibrinogensubstitution bei einem Fibrinogenmangel $(<1,0 \mathrm{~g} / \mathrm{l})$ empfohlen [4]. Die präinterventionelle Transfusion von Thrombozytenkonzentraten oder Fresh Frozen Plasma ist generell nicht empfohlen [5].

\section{Risiken ((Ü2))}

Allgemein kann eine Aszitespunktion als risikoarm betrachtet werden [6,7]. Als häufigste Komplikation wird die Aszitesleckage erwähnt, dies jedoch vor allem, wenn bei der Punktion grosslumige Kanülen, grosse Hautinzisionen oder nicht die empfohlen «Z-Durchstichtechnik» angewendet werden. Schwere Blutungskomplikationen oder Darmperforation sind sehr selten [8]. In einer prospektiven Studie mit 1100 therapeutischen Parazentesen traten keine Blutungskomplikationen auf, trotz Thrombozytopenie (54\% $<50$ 000/ $/$ l) und erhöhtem INR (75\%>1,5 und 26,5\% >2,0) [9]. Folglich stellen auch tiefe Thrombozytenwerte $(<50000 / \mu l)$ und ein erhöhter INR $(>1,5)$ keine Kontraindikation dar. Weitere seltene Komplikationen sind Niereninsuffizienz und Enzephalopathie.

\section{Kontraindikationen ((Ü2))}

Relative Kontraindikationen sind ein unkooperativer Patient, floride Hautinfektion im Bereich der Punktionsstelle, eine Schwangerschaft, massiv dilatierte Darmschlingen oder ausgeprägte Organomegalie. Wenn klinisch eine nachweisbare Fibrinolyse (grosse Ekchymosen oder Hämatome) besteht oder eine disseminierte intravasale Gerinnungsstörung vorliegt, sollte auf eine Punktion verzichtet werden

\section{Durchführung ((Ü2))}

\section{Material ((Ü3))}

Vor der Durchführung der Intervention sollte das Material vorbereitet werden. Es werden Desinfektionsmittel, sterile Kompressen, sterile Handschuhe, ein steriles Lochtuch, Haut-Markierstift, Lidocain 1\%, eine $10 \mathrm{ml}$ Spritze, ein Skalpell (Klinge Nr. 11) und eine 22 Gauge Punktionsnadel benötigt. Zur Drainage empfiehlt es sich, ein für Aszitesdrainage entwickeltes, handelsübliches Punktionsset zu verwenden. Dieses enthält ein Punktionsgerät, das einen Drainagekatheter (14 Gauge) mit einem Punktionsmandrin (teils mit stumpfem, versenkbarem Obturator im Zentrum, um Organverletzungen zu vermeiden) enthält. Über diesen wird nach erfolgreicher Punktion der Drainagekatheter eingeführt. Zudem enthält das Set eine $50 \mathrm{ml}$ Spritze und einen Schlauch je mit Luer-Lock, einen Drei-Wege-Hahn und einen Sekretbeutel (2000 ml).

\section{Lagerung des Patienten ((Ü3))}

Der Patient sollte in Rückenlage mit leicht erhöhtem Kopfteil positioniert werden. Dies bewirkt, dass die luftgefüllten Darmschlingen vom Aszites nach oben gedrängt werden.

\section{Punktionsstelle (mit oder ohne Ultraschall) ((Ü3))}

Früher wählte man die Medianlinie, halber Weg zwischen Umbilikus und Symphyse, als Punktionsstelle, weil sie nicht vaskularisiert ist. Heute wird der linke untere Quadrant als Punktionsstelle favorisiert, weil dort häufiger mehr Aszites zu finden ist und die Abdominalwand dünner und daher der Punktionsweg kürzer ist [10]. Diese 
Punktionsstelle liegt lateral von Musculus rectus abdominis und somit lateral von den epigastrischen Gefässen. Die Harnblase sollte vor Punktion entleert werden. Wenn ein Ultraschallgerät verfügbar ist, dann ist, gerade bei wenig Aszites, die sonographisch-kontrollierte Punktion zu empfehlen. Mit dem Linearultraschall lassen sich optional auch gleich die epigastrischen Gefässe lokalisieren, die es zwingend zu umgehen gilt. Obwohl der Vorteil der ultraschall-kontrollierten gegenüber der konventionellen (klinischen) Punktionsmethode formal nicht evident ist [11], so zeigt sich, dass im Praxisalltag, gerade bei adipösen oder mehrfach voroperierten Patienten, der Ultraschall hilfreich ist. Zudem ist der Ultraschall eine einfache und vielerorts verfügbare Methode.

\section{Vorgehen ((Ü3))}

Vor dem Eingriff ist der Patient ausführlich über die Prozedur aufzuklären und eine Einverständniserklärung sollte vorliegen. Schon vor der Punktion muss festgelegt werden, welche Parameter im Punktat bestimmt und die entsprechenden Probengefässe bereitgestellt werden. Die Punktionsstelle mit einem Marker kennzeichnen. Mit sterilen Handschuhen erfolgt die grosszügige Desinfektion der Haut an der markierten Stelle. Auf das Tragen eines sterilen Mantels und Kopfhaube kann verzichtet werden, jedoch wird empfohlen einen Mundschutz zu tragen. Das sterile Lochtuch wird über die Punktionsstelle gelegt. Senkrecht zur Haut wird die betreffende Region mit einer feinen Nadel mit dem Lokalanästhetikum infiltriert. Die tieferen Gewebeabschnitte im Verlauf des potenziellen Stichkanals werden durch vorsichtiges Vorschieben der Nadel anästhesiert. Durch wiederholte Aspiration alle paar Millimeter wird geprüft, ob das Peritoneum schon passiert ist. Wenn Aszites aspiriert werden kann, sollte zusätzliches Lokalanästhetikum gegeben werden, um das schmerzempfindliche parietale Peritoneum zu anästhesieren. 5-10 ml Lidocain 1\% reichen üblicherweise.

Nach einem kleinen Hautschnitt mit dem spitzen Skalpell kann die Punktionsnadel (Gauge 14) eingeführt werden. Zur Vermeidung eines postpunktuellen Asziteslecks empfiehlt es sich, die Haut sanft (ca. $2 \mathrm{~cm}$ ) nach kaudal zu ziehen und so die Haut gegenüber dem tieferem Gewebe zu verschieben (Z-Durchstichtechnik) oder einen schrägen Einstich im 45-Winkel zu wählen. Beim Durchtritt durchs Peritoneum wird ein plötzlicher Widerstandsverlust verspürt, durch Aspiration von Aszites wird die intraperitoneale Lage bestätigt. Nun kann der Drainagekatheter über die Kanüle bis zum Anschlag eingeführt und die Kanüle entfernen entfernt werden. Über den Drei-Wege-Hahn muss der Schlauch mit dem Beutel angeschlossen werden. Für die diagnostische Analyse wird nun über den Drei-Wege-Hahn mit der 50 ml Spritze Aszites entnommen. Danach lässt man den Aszites passiv ablaufen durch tiefer hängen des Beutels, z.B. am Patientenbett. Zum Ende der Drainage kann der Katheter entfernt werden, dies ist meist nach weniger als zwei Stunden der Fall. Auf keinen Fall sollte der Katheter über Nacht belassen werden. Beim Entfernen der Drainage verschliesst sich der Stichkanal spontan indem die durchdrungenen Schichten sich wieder in die ursprüngliche Position zurückverschieben (ZDurchstichtechnik). So kann ein Aszitesleck praktisch immer vermieden werden. Ein Pflaster an der Punktionsstelle wird angebracht und dem Patienten empfohlen, sich auf die nicht-punktierte Seite zu drehen, dies beugt ebenfalls eine Aszitesleckage vor [12].

\section{Gabe von Humanalbumin ((Ü3))}

Nach einer therapeutischen (grossvolumigen) Parazentese kann es zu Hyponatriämie, Niereninsuffizienz und Enzephalopathie kommen. Um dies zu vermeiden, gibt es verschiedene Studien, die untersucht haben, ob die Gabe von Plasmaexpander oder Humanalbumin während der Parazentese dies eher zu verhindern mag, als wenn physiologische Kochsalzlösung verabreicht wird. In einer Metaanalyse [8] fanden sich keine signifikanten Unterschiede hinsichtlich Morbidität oder Mortalität. Aufgrund noch ungenügender Daten kann jedoch nicht gesagt werden, dass die Zugabe von Plasmaexpander gegenüber physiologischer Kochsalzlösung keine Vorteile bringt und bleibt somit kontrovers diskutiert. Experten empfehlen bei grossvolumigen Aszitesdrainagen von mehr als fünf Liter Humanalbumin zu substituieren. Im Allgemeinen werden pro Liter entfernten Aszites 8-10 g Humanalbumin verabreicht $[13,14]$. 
Bei der Behandlung von Patienten mit spontanbakterieller Peritonitis hingegen gibt es Evidenz, dass die Gabe von Humanalbumin in Kombination mit Ceftriaxon gegenüber einer Therapie mit Ceftriaxon alleine, das Auftreten eines hepatorenalen Syndroms (Typ 1) und die Mortalität signifikant vermindert [15]. Insbesondere bei Patienten mit Serumkreatinin $\geq 88 \mu \mathrm{mol} / \mathrm{L}$ (Norm 62-106 $\mu \mathrm{mol} / \mathrm{l}$ ) und Bilirubin $\geq 68 \mu \mathrm{mol} / \mathrm{l}$ (Norm $\leq 21 \mu \mathrm{mol} / \mathrm{l})$. Deshalb ist neben der antibiotischen Therapie mit einem Cephalosporin der dritten Generation auch die Gabe von Humanalbumin (1,5 g/kg Körpergewicht am Tag 1 bei Diagnose und $1 \mathrm{~g} / \mathrm{kg}$ an Tag 3) indiziert. 
Analyse und Interpretation der Resultate/differenzialdiagnostische Überlegungen ((Ü1))

Folgender Algorithmus (zusammengefasst in Tabelle 2) soll helfen festzulegen, wann welche Parameter sinnvoll und kosteneffektiv zu bestimmen sind.

\section{((Hier etwa Tabelle 2))}

\section{Zellzahl, Zelldifferenzierung und mikrobiologische Asziteskultur ((Ü2))}

Bei unkompliziertem Aszites infolge Leberzirrhose reicht die Bestimmung der Zellzahl (und -Differenzierung). Wenn der Verdacht auf eine spontanbakterielle Peritonitis besteht, sollten zusätzlich Blutkulturflaschen mit Aszites inokuliert werden. Zur Diagnose einer spontanbakteriellen Peritonitis gilt gemeinhin ein Grenzwert von $\geq 250$ neutrophile Granulozyten (PMN) pro Mikroliter im Aszites, in Abwesenheit einer anderen, intraabdominellen, behandlungsbedürftigen Infektion. Falls keine Zelldifferenzierung verfügbar ist, spricht man von einer SBP bei $\geq 500$ Leukozyten/ $\mu$ l. Bakterielles Wachstum findet sich nur in etwa $40 \%$ des Aszites mit PMN $\geq 250 / \mu l$ [16]. Die tiefe Rate an positiven Asziteskulturen ist wahrscheinlich auf die tiefe Bakterienkonzentration im Aszites zurückzuführen, verglichen mit Infektionen anderer Körperflüssigkeiten (z.B. Urin). Bei Verdacht auf eine SBP ist ein rascher Beginn einer empirischen antibiotischen Therapie mit Cephalosporinen der dritten Generation oder Amoxicillin-Clavulansäure indiziert. Eine SBP geht mit einer hohen Mortalität einher [2]. Eine Sonderform stellt der Bakteraszites dar, definiert durch eine positive Asziteskultur bei PMN $\leq 250 / \mu \mathrm{L}$ ohne Hinweise für eine lokale oder systemische Infektion. Hier bedarf es einer Wiederholung der Aszitespunktion. Eine antibiotische Therapie ist empfohlen wenn dann die PMN $\geq 250 / \mu \mathrm{L}$, die Kultur positiv oder Nachweis einer lokalen oder systemischen Infektion besteht.

\section{Albumin ((Ü2))}

Schon lange wurde gezeigt, dass sich der Serum-Aszites Albumin-Gradient (SAAG) besser eignet als das Exsudat-Transsudat-Modell in der Differenzialdiagnose des Aszites, wenn es um portale Hypertonie geht [17]. Aus der Differenz von Albumin im Serum zu Albumin im Aszites wird der SAAG berechnet. Ein SAAG von $\geq 11 \mathrm{~g} / \mathrm{l}$ spricht für eine portale Hypertonie als Ursache des Aszites. Umgekehrt spricht ein tiefer Gradient $(<11 \mathrm{~g} / l)$ gegen eine portale Hypertonie und andere Ursachen für Aszites müssen gesucht werden (Tab. 1).

\section{Gesamtprotein ((Ü2))}

Bei erhöhtem SAAG (>11 g/l) kann das Gesamtprotein im Aszites zur weiteren Differenzierung herangezogen werden. Liegt das Gesamtprotein $<25 \mathrm{~g} / \mathrm{l}$, spricht dies für Aszites aufgrund einer Leberzirrhose. Liegt das Gesamtprotein dagegen $>25 \mathrm{~g}$, weist dies auf eine kardiale Ursache, im Sinne einer Rechtsherzinsuffizienz hin. Bei tiefem SAAG ( $<11 \mathrm{~g} / \mathrm{l})$ und tiefem Gesamtprotein $(<25 \mathrm{~g} /)$ ist die Konstellation typisch für renalen Aszites bei nephrotischer Proteinurie. Patienten mit sehr tiefem Gesamtprotein haben ein erhöhtes Risiko für eine SBP [18].

\section{Zytologie ((Ü2))}

Die oben definierten Grenzwerte ( $\geq 250 \mathrm{PMN} / \mu \mathrm{L}$ ) helfen zur Unterscheidung von infiziertem und so genannt sterilem Aszites bei portaler Hypertonie/Leberzirrhose. Es hilft jedoch nicht zur Unterscheidung von infiziertem Aszites und malignem Aszites. Deshalb sollte bei der Erstdiagnose von Aszites auch eine zytologische Untersuchung erfolgen - hierzu sollte möglichst viel Untersuchungsmaterial verwendet werden, dies erhöht die Sensitivität der zytologischen Untersuchung. Der Nachweis von malignen Zellen im Aszites definiert eine Peritonealkarzinose. 


\section{Mykobakteriologie ((Ü2))}

Eine mykobakterielle Kultur gehört nicht zur Routineuntersuchung. Bei Patienten mit hohem Risiko für tuberkulöse Peritonitis infolge kürzlicher Migration aus einem Endemiegebiet oder durch erworbene Immundefizienz (z.B. HIV) sollten an eine tuberkulöse Peritonitis gedacht werden. Die Suche nach säurefesten Stäbchen hat eine sehr schlechte Sensitivität. Gegebenenfalls ist bei hohem Verdacht eine diagnostische Laparoskopie und eine Biopsie von Peritoneum zur Kultur und PCR (Polymerase Kettenreaktion) durchzuführen [19].

Folgende Parameter haben in der Diagnostik bei Zirrhosepatienten und Aszites keinen Stellenwert, sie werden bei spezifischer Fragestellung in einem anderen klinischen Kontext zur Differenzialdiagnose herangezogen:

\section{Amylase ((Ü3))}

Bei stark erhöhter Amylase im Aszitespunktat (insbesondere im Verhältnis zur Serum-Amylase) liegt meist eine Pankreatitis vor. Es kann aber auch Ausdruck einer Dünndarmperforation sein.

\section{Triglyzeride ((Ü3))}

Bei Drainage von milchig-trübem Aszites ist die Bestimmung von Triglyzeriden im Punktat empfohlen. Bei erhöhten Triglyzeridwerte (definiert als $>2,3 \mathrm{mmol} / \mathrm{l}$ ) spricht man von chylösem Aszites. Dieser kann durch tumoröse Abflussbehinderung des Ductus thoracicus entstehen oder es muss an ein Leck der Lymphgefässe gedacht werden.

\section{Bilirubin ((Ü3))}

Stark ikterische Patienten haben braunen Aszites aufgrund des Bilirubins. Die Konzentration im Aszites entspricht ca. der Hälfte der Serumkonzentration. Sollte das Bilirubin im Aszites höher sein als im Serum, spricht dies für ein biliäre Leckage.

\section{Cholesterin, pH und Laktat ((Ü3))}

Diese Parameter haben sich in der Aszitesdiagnostik als nicht hilfreich erwiesen $[20,21]$. 
Therapie und Prognose ((Ü1))

Jeder neuaufgetretene Aszites sollte punktiert und die Ursache geklärt werden. Die Durchführung ist einfach und schwere Komplikationen sind sehr selten. Eine sonographisch kontrollierte Punktion ist zu empfehlen. Das Auftreten von Aszites im Rahmen einer bekannten Leberzirrhose ist ein prognostisch schlechtes Zeichen. Das Vorliegen einer spontanbakteriellen Peritonitis geht mit einer hohen Mortalität einher und bedarf daher einer frühen Erkennung und Therapie. Patienten mit Leberzirrhose und Aszites sollten hinsichtlich einer Lebertransplantation evaluiert werden. 


\section{Fallbericht zu Aszites: Abdominale Parazentese}

Y

A Anamnese des Patienten ((Ü1))

Weg zur Diagnose/Kommentar ((Ü1))

\begin{tabular}{|l|l|lrr}
\hline Die Zuweisung des 62-jährigen Patienten erfolgte zur & Die Müdigkeit und die Zunahme des \\
Abklärung einer Lebertransplantation bei vermutlich & Bauchumfanges mit Druckgefühl sind klassische \\
äthyltoxischer Leberzirrhose mit progredienter klinischer & Beschwerden bei Aszites. Der Gewichtsverlust \\
Verschlechterung. Der Patient berichtete über Müdigkeit & passt gut zum Bild der Leberzirrhose, meist im \\
und markantem Gewichtsverlust in den letzten Monaten & Zusammenhang mit Appetitlosigkeit und Nausea. \\
trotz signifikanter Zunahme des Bauchumfanges mit & \\
Druckgefühl. &
\end{tabular}

1

Befunde des Patienten ((Ü1))

Weg zur Diagnose/Kommentar ((Ü1))

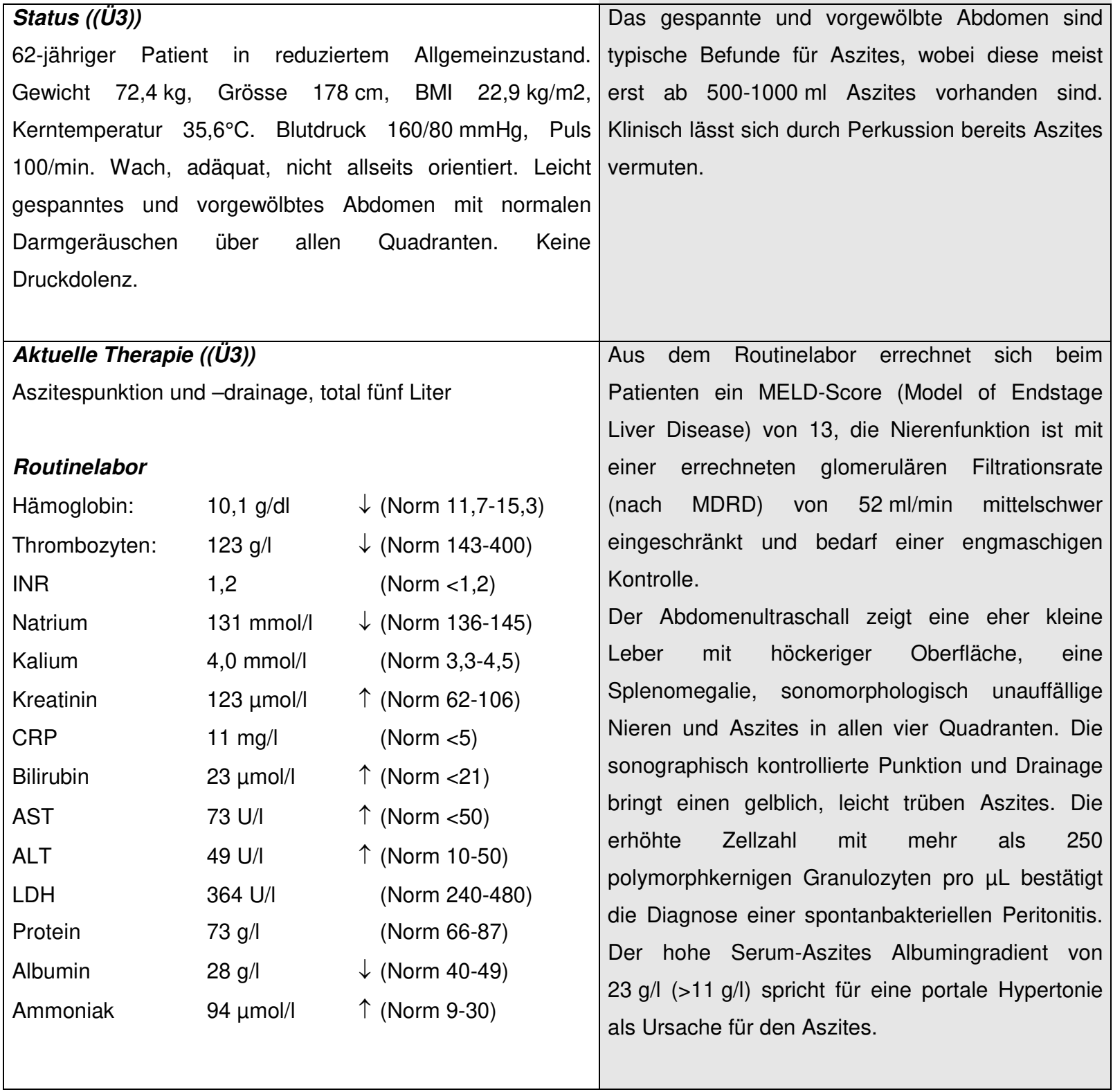




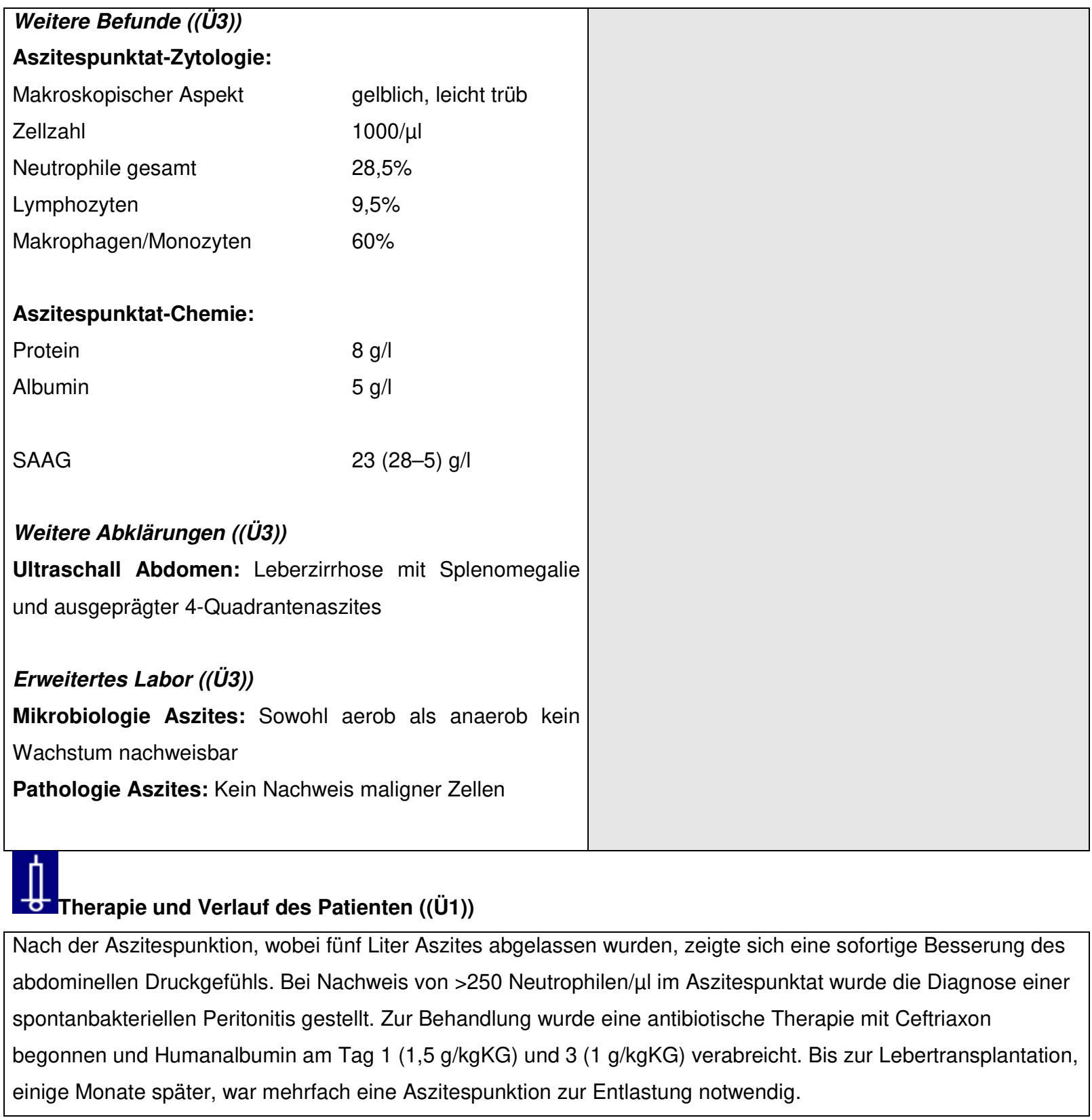




\section{Fragen zu Aszites: Abdominale Parazentese}

\section{Frage 1}

Die häufigste Ursache für Aszites ist (Einfachauswahl, 1 richtige Antwort)
a) Pankreatitis.
b) Leberzirrhose.
c) Peritonealkarzinose.
d) Rechtsherzinsuffizienz.
e) Niereninsuffzienz.

\section{Frage 2}

Eine Aszitespunktion wird durchgeführt, (Einfachauswahl, 1 richtige Antwort)

a) nur wenn der INR $<1,5$ ist, da es sonst zu vermehrten Blutungskomplikationen kommt.

b) nur wenn die Thrombozytenzahl $>100 \mathrm{~g} / \mathrm{l}$ ist.

c) nur wenn bei erhöhtem INR zuvor Fresh Frozen Plasma (FFP) verabreicht wurde.

d) nur wenn der Patient nüchtern ist.

e) Keine der obengenannten Aussagen trifft zu.

\section{Frage 3}

Folgender Wert spricht am zutreffendsten für eine portale Hypertonie als Ursache für Aszites:

(Einfachauswahl, 1 richtige Antwort)

a) Tiefes Cholesterin im Aszites

b) Tiefe Zellzahl im Aszites

c) Serum-Aszites Albumingradient $>11 \mathrm{~g} / \mathrm{l}$

d) Serum-Aszites Albumingradient $<11 \mathrm{~g} / \mathrm{l}$

e) Das Laktat im Aszites ist kleiner als im Serum.

\section{Frage 4}

Eine spontanbakterielle Peritonitis (Einfachauswahl, 1 richtige Antwort)

a) wird diagnostiziert bei einer Zellzahl von $>250 / \mu$ im Aszites.

b) wird mittels Computertomographie diagnostiziert.

c) hat eine gute Prognose und heilt spontan aus.

d) verläuft nie asymptomatisch.

e) ist definiert durch Nachweis von $\geq 250$ polymorphkernigen Granulozyten/ $\mu$ l Aszites.

\section{Frage 5}

Bei grossvolumiger Aszitesdrainage ( $>5$ Liter) (Einfachauswahl, 1 richtige Antwort)

a) ist die Substitution von $10 \mathrm{~g}$ Humanalbumin pro drainierten Liter empfohlen.

b) ist zur symptomatischen Therapie bei therapierefraktärem Aszites indiziert.

c) kann zu Niereninsuffizienz führen.

d) kann ambulant durchgeführt werden.

e) Alle Antworten treffen zu.

\section{Korrespondenzadresse}


Dr. med. Stephan Bosshard

Klinik und Poliklinik für Innere Medizin

Universitätsspital Zürich

Rämistrasse 100

8091 Zürich

stephan.bosshard@usz.ch

\section{Autoren}

Klinik und Poliklinik für Innere Medizin ${ }^{1}$, Klinik für Gastroenterologie und Hepatologie ${ }^{2}$, UniversitätsSpital Zürich ${ }^{1}$ Dr. med. S. Bosshard, ${ }^{2}$ Prof. Dr. med. B. Müllhaupt 
DOI 10.1024/1661-8157/a000955

((PX12_Rubrik CME-Antworten))

\section{Antworten zu den Fragen zur Aszitespunktion aus PRAXIS}

\section{Frage 1}

\section{Richtig ist Antwort b).}

Ad b): In Europa haben drei von vier Patienten, die sich mit Aszites vorstellen eine Leberzirrhose.

Ad a) und c) bis e): Alle anderen Ursachen sind deutlich seltener.

\section{Frage 2}

Richtig ist Antwort e).

Ad a) bis c): Das Blutungsrisiko ist auch bei erhöhtem INR und bei Thrombozytopenie sehr gering. Eine Substitution mit Fresh Frozen Plasma oder Thrombozytenkonzentrat vor einer Aszitespunktion ist nicht indiziert. Es wird empfohlen ein vorhandener Fibrinogenmangel $(<1,0 \mathrm{~g} / \mathrm{l})$ zu substituieren.

Ad d): Nüchternheit ist keine Voraussetzung bei einer Aszitespunktion. Eine Entleerung der Harnblase vor der Punktion ist hingegen zu empfehlen.

\section{Frage 3}

\section{Richtig ist Antwort c).}

Ein Serum-Aszites-Albumingradient von $>11 \mathrm{~g} / \mathrm{l}$ spricht für das Vorliegen einer portalen Hypertonie.

Ad a) und e): Cholesterin und Laktat sind in der Differenzierung der Ursache von Aszites nicht hilfreich und sollten nicht bestimmt werden.

Ad b): Die Zellzahl hat keinen Aussagewert bezüglich portaler Hypertension.

\section{Frage 4}

\section{Richtig ist Antwort e).}

Eine spontanbakterielle Peritonitis liegt vor bei Nachweis von > 250 polymorphkernigen Granulozyten/ $\mu$ l Aszites. Ad a) bis d): Eine spontanbakterielle Peritonitis verläuft nicht selten asymptomatisch und hat im Zusammenhang einer Leberzirrhose eine hohe Mortalität. Sie sollte entsprechend den Guidelines mit Albuminsubstitution am Tag 1 (1,5 g/kgKG) und Tag 3 (1 g/kgKG) und nach Abnahme von Kulturen mit einem Cephalosporin der dritten Generation behandelt werden. Eine Zellzahl von >250/ $\mu \mathrm{l}$ im Aszites sollte weiter differenziert werden.

\section{Ad b): ((Autor: hier fehlt die Erklärung))}

\section{Frage 5}

\section{Richtig ist Antwort e).}

Alle Aussagen sind korrekt, somit ist Antwort e) richtig.

$\mathrm{Ad} \mathrm{a)} \mathrm{und} \mathrm{c):} \mathrm{Bei} \mathrm{grossvolumiger} \mathrm{Aszitespunktion} \mathrm{ist} \mathrm{die} \mathrm{Substitution} \mathrm{von} \mathrm{Humanalbumin} \mathrm{mit} 10 \mathrm{~g}$ pro abgelassenem Liter Aszites empfohlen, um eine (prärenale) Nierenfunktionsverschlechterung vorzubeugen. Ad b) und d): Bei therapierefraktärem Aszites sollte die Einlage eines transjugulären porto-systemischen Shunts (TIPS) evaluiert werden, ansonsten ist die wiederholte, therapeutische Aszitespunktion die einzige Alternative und kann gut ambulant erfolgen. 


\section{Bibliographie}

1. Benvegnù L, Gios $M$, Boccato $S$, Alberti $A$ : Natural history of compensated viral cirrhosis: a prospective study on the incidence and hierarchy of major complications. Gut 2004; 53: 744-749.

2. Thuluvath PJ, Morss S, Thompson R: Spontaneous bacterial peritonitis--in-hospital mortality, predictors of survival, and health care costs from 1988 to 1998. Am J Gastroenterol 2001; 96: 1232-1236.

3. Borzio M, Salerno F, Piantoni L, Cazzaniga M, Angeli P, Bissoli F, et al.: Bacterial infection in patients with advanced cirrhosis: a multicentre prospective study. Dig Liver Dis 2001; 33: 41-48.

4. O'shaughnessy DF, Atterbury C, Bolton Maggs P, Murphy M, Thomas D, Yates S, et al.: Guidelines for the use of fresh-frozen plasma, cryoprecipitate and cryosupernatant. Br J Haematol 2004; 126: 11-28.

5. Warmuth M, Mad P, Wild C: Systematic review of the efficacy and safety of fibrinogen concentrate substitution in adults. Acta Anaesthesiol Scand 2011; doi: 10.1111/j.1399-6576.2011.02586.x.

6. Runyon BA: Paracentesis of ascitic fluid. A safe procedure. Arch Intern Med 1986; 146: 2259-2261.

7. De Gottardi A, Thevenot T, Spahr L, Morard I, Bresson-Hadni S, Torres F, et al.: Risk of complications after abdominal paracentesis in cirrhotic patients: a prospective study. Clin Gastroenterol Hepatol 2009; 7: 906-909.

8. Wong CL, Holroyd-Leduc J, Thorpe KE, Straus SE: Does this patient have bacterial peritonitis or portal hypertension? How do I perform a paracentesis and analyze the results? JAMA 2008; 299: 1166-1178.

9. Grabau CM, Crago SF, Hoff LK, Simon JA, Melton CA, Ott BJ, et al.: Performance standards for therapeutic abdominal paracentesis. Hepatology 2004; 40: 484-488.

10. Sakai $\mathrm{H}$, Sheer TA, Mendler MH, Runyon BA: Choosing the location for non-image guided abdominal paracentesis. Liver Int 2005; 25: 984-986.

11. Nazeer SR, Dewbre H, Miller AH: Ultrasound-assisted paracentesis performed by emergency physicians vs the traditional technique: a prospective, randomized study. Am J Emerg Med 2005; 23: 363-367. 12. Thomsen TW, Shaffer RW, White B, Setnik GS: Videos in clinical medicine. Paracentesis. N Engl J Med 2006; 355: e21.

13. Wang F, Wang BY: Progress in the diagnosis and treatment of ascites in cirrhosis: introduction of EASL clinical practice guidelines on management of ascites in cirrhosis. Zhonghua Gan Zang Bing Za Zhi 2010; 18: 951-954.

14. Runyon BA: Management of adult patients with ascites due to cirrhosis: an update. Hepatology 2009; 49: 2087-2107.

15. Sort P, Navasa M, Arroyo V, Aldeguer X, Planas R, Ruiz-del-Arbol L, et al.: Effect of intravenous albumin on renal impairment and mortality in patients with cirrhosis and spontaneous bacterial peritonitis. $\mathrm{N}$ Engl J Med 1999; 341: 403-409.

16. Rimola A, Garcia-Tsao G, Navasa M, Piddock LJ, Planas R, Bernard B, et al.: Diagnosis, treatment and prophylaxis of spontaneous bacterial peritonitis: a consensus document. International Ascites Club. J Hepatol 2000; 32: 142-153.

17. Runyon BA, Montano AA, Akriviadis EA, Antillon MR, Irving MA, McHutchinson JG: The serumascites albumin gradient is superior to the exudate-transudate concept in the differential diagnosis of ascites. Ann Intern Med 1992; 117: 215-220.

18. Runyon BA: Low-protein-concentration ascitic fluid is predisposed to spontaneous bacterial peritonitis. Gastroenterology 1986; 91: 1343-1346.

19. Al Karawi MA, Mohamed AE, Yasawy MI, Graham DY, Shariq S, Ahmed AM, et al.: Protean manifestation of gastrointestinal tuberculosis: report on 130 patients. J Clin Gastroenterol 1995; 20: $225-232$. 20. Runyon BA, Antillon MR: Ascitic fluid $\mathrm{pH}$ and lactate: insensitive and nonspecific tests in detecting ascitic fluid infection. Hepatology 1991; 13: 929-935. 
21. Runyon BA: Malignancy-related ascites and ascitic fluid «humoral tests of malignancy». J Clin Gastroenterol 1994; 18: 94-98. 
Tab 1: Häufigste Ursachen von Aszites

\begin{tabular}{|ll|}
\hline Hepatisch & $\begin{array}{l}\text { Leberzirrhose } \\
\text { Alkoholische Hepatitis } \\
\text { Leberversagen }\end{array}$ \\
\hline Renal & Nephrotisches Syndrom \\
\hline Kardial & $\begin{array}{l}\text { Rechtsherzinsuffizienz } \\
\text { Perikarditis }\end{array}$ \\
\hline Pankreatogen & Pankreatitis \\
\hline Maligne & Peritonealkarzinose \\
\hline Vaskulär & Budd-Chiari \\
& Pfortaderthrombose \\
\hline Infektiös & Spontanbakterielle Peritonitis \\
& Tuberkulose \\
\hline Traumatisch & Hämorraghie verletzer innerer Organe \\
& Postoperatives lymphatische Leckage \\
\hline Infektiös & Spontanbakterielle Peritonitis \\
& Tuberkulose \\
\hline
\end{tabular}


Tab. 2: Parameter in der Aszitesdiagnostik

\begin{tabular}{|l|l|l|}
\hline Routine Diagnostik & Erweiterte Diagnostik & Nicht hilfreich \\
\hline \hline Zellzahl und Differenzierung & Zytologie & $\mathrm{pH}$ \\
Asziteskultur & Mykobakteriologie (PCR, Kultur) & Cholesterin \\
Albumin & Amylase & Laktat \\
Gesamtprotein & Triglyzeride & \\
$(($ Autor: fehlt hier was?)) & Bilirubin & \\
\hline
\end{tabular}

